Pecvnia, 1 (2005), pp. 13-29

\title{
Barómetro industrial LEON 50
}

\author{
Javier Álvarez Martínez \\ Julio Lago Rodríguez \\ David Pacho Santos
}

El Barómetro Industrial LEON 50 es un trabajo de investigación que tiene como objetivo esencial identificar características patrimoniales, financieras y económicas de una muestra de empresas que actúan dentro de la industria leonesa. El soporte de este estudio empírico es la información contable anual auditada que depositan en el Registro Mercantil las empresas integrantes de la muestra, recogida básicamente en el balance de situación, la cuenta de pérdidas y ganancias y el cuadro de financiación referidos al bienio 2001-02. En esencia, el Barómetro Industrial LEON 50 pretende ser un avance en el análisis, diagnóstico y mejor conocimiento de la industria regional, aportando una visión actualizada sobre estrategias y pautas de actuación empresarial que serán objeto de observación en períodos venideros.

Palabras clave: Análisis contable. Estudios industriales. Economía leonesa.
The Leon 50 Industrial Barometer is a work of research which has as its main objective to identify financial, economical and capital resource characteristics of a sample of companies within the ambit of Leonese industry. The basis of this empiric study is the annual audited accounting information which is deposited in the Business Register by the business composing the sample. The information is taken basically from the balance-sheet, the profit and loss account and the financial table for the period 2001-02. In essence, the Leon 50 Industrial Barometer aims to provide a development in the analysis, diagnosis and greater awareness of the Leonese economy, offering a contemporary view of strategies and business guidelines which will be the subject of the future observation.

Key words: Accountant Analysis. Industry Studies. Leonese Economy. 


\section{LEON 50}

\section{OBJETIVOS Y METODOLOGÍA DEL BARÓMETRO INDUSTRIAL}

El Barómetro Industrial LEON 50, en su segundo informe, es un trabajo de investigación que da continuidad al que se presentaba en junio de 2003 tomando como referencia una muestra de 40 empresas. Básicamente pretende ser un observatorio de la industria leonesa que tiene por objetivo identificar la vitalidad y las características patrimoniales, financieras y económicas más significativas de una muestra de empresas que actúan dentro de este sector económico.

Para consumar este propósito se confecciona un barómetro selectivo constituido por 50 empresas industriales de propiedad privada (LEON 50), tanto de capital leonés, español como extranjero, escogidas atendiendo a criterios de relevancia productiva y geográfica.

Con el fin de englobar múltiples casuísticas industriales, la configuración de la muestra opta por incluir compañías que realizan muy distintos grados de transformación sobre las materias primas adquiridas a otras empresas.

Actúa como base o soporte de este estudio empírico la información contable anual auditada que depositan en el Registro Mercantil las empresas integrantes de la muestra, recogida básicamente en el balance de situación, la cuenta de resultados y el cuadro de financiación referidos al bienio 2001-02.

Se ha tomado como fecha de inicio de la investigación el año 2001 por ser un año relevante desde el punto de vista económico: antesala a la plena implantación del euro. Así mismo, es el período de tiempo más actual para el que se dispone de información empresarial completa accesible en las bases de datos de los Registros Mercantiles.

La confección de este índice selectivo de empresas industriales de la economía leonesa nace con vocación de continuidad, en el sentido de programarse un seguimiento temporal que tiene como finalidad esencial detectar, mediante técnicas de análisis contable, comportamientos empresariales en materia de financiación, equilibrio financiero, rentabilidad, volumen de las inversiones, empleo, tanto fijo como no fijo, y otras variables afines que definen rasgos fundamentales de la gestión de los negocios.

A este respecto, se mantendrá a lo largo del tiempo la identidad de las empresas objeto de análisis, si bien no se descarta 
aumentar de manera progresiva el tamaño de la muestra con el fin de conseguir una mayor representatividad. De hecho, y como ya se deduce de la redacción del punto primero, el informe de 2002 ha incrementado en diez el número de empresas con respecto al estudio de 2001.

En esencia, el Barómetro Industrial LEON 50 pretende ser un avance, un paso más, en el estudio, análisis, diagnóstico y mejor conocimiento de la industria leonesa, aportando una visión actualizada sobre estrategias y pautas de actuación empresarial que serán objeto de observación en períodos venideros. En el anexo que figura al final del trabajo se relacionan los ratios utilizados para efectuar los diagnósticos intertemporales, empleándose como herramienta de apoyo informático para este fin la hoja de cálculo excel.

\section{ESTRUCTURA DEL BARÓMETRO INDUSTRIAL LEON 50}

a) Relación nominal de empresas

1. ACEROS ROLDAN, S.A.

2. ANTIBIÓTICOS, S.A.

3. ALIMENTOS NATURALES, S.A.

4. BAÑEZANA DE FERRALLA, S.L.

5. BA - VIDRIO, S.A.

6. CECINAS PABLO, S.A.

7. CEMENTOS COSMOS, S.A.

8. CEMENTOS TUDELA VEGUIN, S.A.

9. CERÁMICAS DEL NOROESTE, S.A. (CERANOR, S.A.)

10. COMERCIAL VINÍCOLA VILLAFRANQUINA, S.A.

11. CONGELADOS Y DERIVADOS, S.A. (ELMAR)

12. CONSTRUCCIONES METALICAS LOS BLANCOS, S.L.

13. CONSTRUCCIONES Y MONTAJES DEL NOROESTE, S.A. (COMONOR)

14. CRISTALERA INDUSTRIAL, S.A.

15. EMBUTIDOS RODRÍGUEZ, S.L.

16. ENERGIA VIVA, S.A. (ENERVISA)

17. EDITORIAL EVEREST, S.A.

18. FAMINOR S.L.

19. FERRALLA EDUARDO E HIJOS, S.A.

20. FRIGORÍFICOS INDUSTRIALES DEL BIERZO, S.A. (FRIBER)

21. GARNICA PLYWOOD, S.L.

22. GORDONCELLO, S.A.

23. HIJOS DE ANTONIO GASPAR ROSA, S.L.

24. HULLERA VASCO LEONESA, S.A.

25. INDUSTRIAS LACTEAS MANZANO, S.A.

26. KRAFT FOODS ESPAÑA, S.A.

27. LA BALINESA, S.L. 


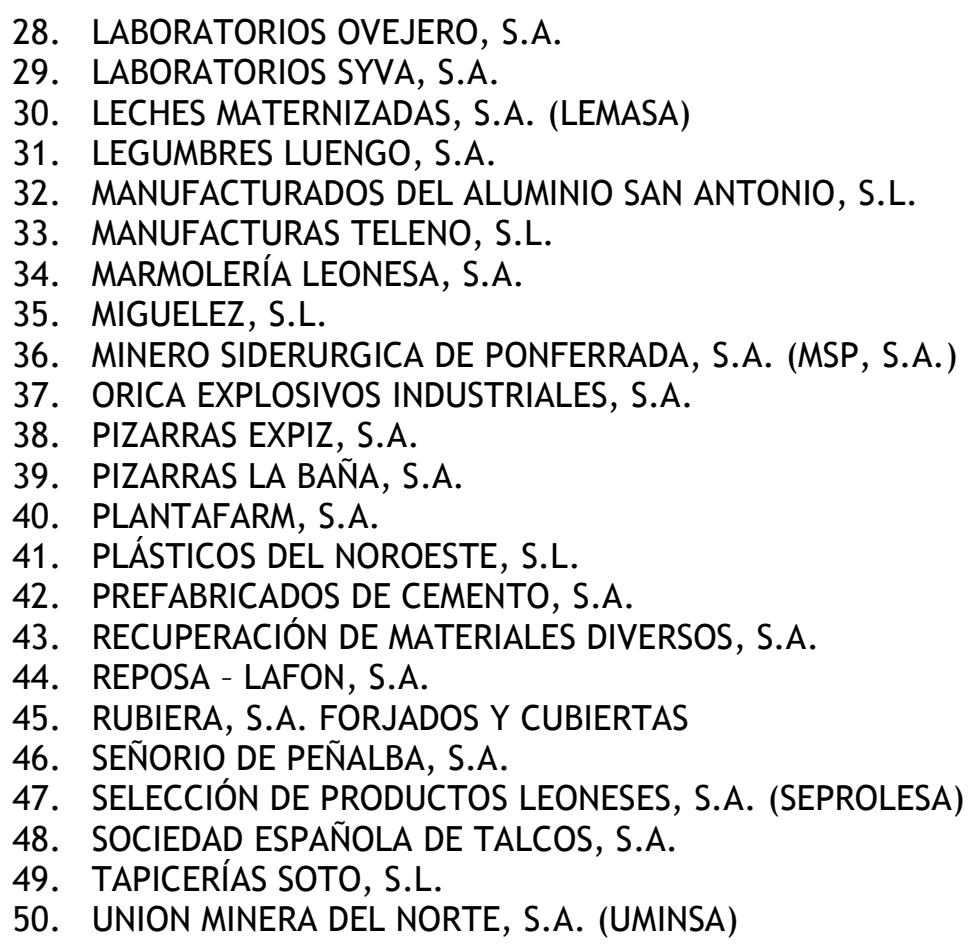

b) Caracterización económica de la muestra

Las magnitudes agregadas representativas del nivel de actividad del conjunto de las cincuenta empresas integrantes de la muestra son las siguientes:

- Plantilla promedio (personal asalariado): 8.362 empleos ( $88 \%$ fijos)

- Cifra de negocio: $1.500 \mathrm{M}$ euros (250.000 M ptas)

- Inversión: 1.880 M euros (312.000 M ptas)

- Nómina (sueldos y salarios): $206 \mathrm{M}$ euros (34.275 M ptas)

- Beneficios: $108 \mathrm{M}$ euros (18.000 M ptas)

C) Clasificación por ramas productivas

Las empresas integrantes de la muestra se han agrupado en once ramas de actividad, primando la presencia de empresas adscritas a la industria de alimentos (1/3 de la muestra) por la abundancia de producciones amparadas por marchamos de calidad (denominación de origen e indicación geográfica protegida). Es, por otra parte, la actividad industrial más extendida dentro de la geografía leonesa. 
INDUSTRIA QUÍMICA (3)

Antibióticos, S.A. (fabricación de penicilinas y derivados)

Laboratorios Ovejero, S.A. (fabricación de productos zoosanitarios)

Laboratorios SYVA, S.A. (fabricación de productos zoosanitarios)

INDUSTRIA DEL CEMENTO (2)

Cementos Cosmos, S.A. (fabricación de cementos)

Cementos Tudela Veguín, S.A. (fabricación de cementos)

INDUSTRIA DEL METAL (11)

Aceros Roldán, S.A. (fabricación de aceros inoxidables)

Miguélez, S.L. (fabricación de cables eléctricos)

Comonor, S.A. (fabricación de estructuras metálicas y componentes eólicos)

Construcciones Metálicas Los Blancos, S.L. (fabricación de estructuras metálicas)

Tapicerías Soto, S.L. (fabricación de mobiliario metálico)

Reposa - Lafon, S.A. (fabricación de depósitos industriales)

Bañezana de Ferralla, S.L. (fabricación de ferrallas)

Ferralla Eduardo e Hijos, S.A. (fabricación de ferrallas).

Manufacturados de Aluminio San Antonio, S.L. (accesorios de aluminio)

Recuperación de Materiales Diversos, S.A. (recuperación de materiales de desecho)

Faminor, S.L. (fabricación de estructuras metálicas)

INDUSTRIA DEL VIDRIO (2)

BA - Vidrio, S.A. (fabricación de vidrio hueco)

Cristalera Industrial, S.A. (fabricación de vidrio plano)

INDUSTRIA TEXTIL (1)

Manufacturas Teleno, S.L. (fabricación de corsetería)

INDUSTRIA ARTES GRÁFICAS (1)

Editorial Everest, S.A. (impresión y edición de libros) 
INDUSTRIA DE ALIMENTOS (16)

Kraft Foods España, S.A. (fabricación de productos lácteos)

Congelados y Derivados, S.A. (Elmar) (elaboración de alimentos congelados)

Alimentos Naturales, S.A. (fabricación de conservas vegetales)

Gordoncello, S.A. (elaboración de vinos)

Cecinas Pablo, S.A. (fabricación de embutidos)

Legumbres Luengo, S.A. (fabricación de conservas vegetales)

Leches Maternizadas, S.A. (fabricación de alimentos animales)

Plantafarm, S.A. (elaboración de hierbas y plantas medicinales)

Comercial Vinícola Villafranquina, S.A. (elaboración de vinos)

Embutidos Rodríguez, S.L. (fabricación de embutidos)

Industrias Lácteas Manzano, S.A. (fabricación de productos lácteos)

Selección de Productos Leoneses, S.A. (fabricación de conservas vegetales)

Frigoríficos Industriales del Bierzo, S.A. (fabricación de embutidos)

Señorío de Peñalba, S.A. (elaboración de vinos)

Energía Viva, S.A. (producción cárnica de calidad en régimen intensivo)

La Balinesa, S.L. (fabricación de ahumados)

\section{INDUSTRIA DEL CARBÓN (3)}

Minero Siderúrgica de Ponferrada, S.A. (producción de carbón termoeléctrico)

Hullera Vasco-Leonesa, S.A. (producción de carbón termoeléctrico)

Unión Minera del Norte, S.A. (producción de carbón termoeléctrico)

\section{INDUSTRIA DE LOS MATERIALES DE CONSTRUCCIÓN (8)}

Cerámicas del Noroeste, S.A. (fabricación de ladrillos)

Rubiera, S.A. (fabricación de ladrillos, forjados y cubiertas)

Marmolería Leonesa, S.A. (elaboración de piedra natural)

Orica Explosivos Industriales, S.A. (fabricación de explosivos para obra civil)

Pizarras Expiz, S.A. (elaboración de pizarra)

Pizarras La Baña, S.A. (elaboración de pizarra)

Prefabricados de Cementos, S.A. (fabricación de estructuras de cemento)

Plásticos del Noroeste, S.A. (fabricación de puertas, ventanas y persianas)

INDUSTRIA DE LA MADERA (2)

Garnica Plywood, S.L. (fabricación de tableros contrachapados)

Hijos de Antonio Gaspar Rosa, S.L. (fabricación de tableros contrachapados) 
INDUSTRIA DEL TALCO (1)

Sociedad Española de Talcos, S.A. (Luzenac SET) (elaboración de talco)

d) Clasificación por tamaños empresariales

Se han utilizado como criterios de clasificación dos variables de dimensión empresarial, plantilla y cifra de negocios, habitualmente manejadas en el análisis de actividades productivas, distinguiéndose tres formatos: pequeñas, medianas y grandes empresas.

Empresas Pequeñas (18)

Plantilla: $10-50$ trabajadores

Cifra de negocio: 1 - 9,02 M€ (166 - 1.500 M ptas)

Hijos de Antonio Gaspar Rosa, S.L.

Comercial Vinícola Villafranquina, S.A.

Faminor, S.L.

Bañezana de Ferralla, S.L.

Gárnica Plywood, S.L.

Frigoríficos Industriales del Bierzo, S.A. (FRIBER)

Gordoncello, S.A.

Señorío de Peñalba, S.A.

Construcciones Metálicas Los Blancos, S.L.

Industrias Lácteas Manzano, S.A.

Orica Explosivos Industriales, S.A.

Cecinas Pablo, S.A.

Ferralla Eduardo e Hijos, S.A.

La Balinesa, S.L.

Manufacturas de Aluminio San Antonio, S.L.

Plásticos del Noroeste, S.L.

Prefabricados de Cementos, S.A.

Reposa - Lafon, S.A.

Empresas Medianas (22)

Plantilla: 51-250 trabajadores

Cifra de negocio: $9,03-50 \mathrm{M} €(1.501-8.320 \mathrm{M}$ ptas $)$

Cerámicas del Noroeste, S.A. (CERANOR)

Construcciones y Montajes del Noroeste, S.A. (COMONOR) 
Cristalera Industrial, S.A.

Energía Viva, S.A. (ENERVISA)

Editorial Everest, S.A.

Pizarras Expiz, S.A.

Recuperación de Materiales Diversos, S.A.

Leches Maternizadas, S.A. (LEMASA)

Sociedad Española de Talco, S.A. (LUZENAC SET)

Plantafarm, S.A.

Embutidos Rodríguez, S.L.

Rubiera, S.A. Forjados y Cubiertas

Selección de Productos Leoneses, S.A. (SEPROLESA)

Tapicerías Soto, S.L.

Laboratorios SYVA, S.A.

Manufacturas Teleno, S.L.

Marmolería Leonesa, S.L.

Legumbres Luengo, S.A.

Pizarras La Baña, S.A.

Alimentos Naturales, S.A.

Cementos Cosmos, S.A.

Laboratorios Ovejero, S.A.

Empresas Grandes (10)

Plantilla: $>250$ trabajadores

Cifra de negocios: $>50 M €(>8.320$ M ptas)

Antibióticos, S.A.

BA - Vidrio, S.A.

Congelados y Derivados, S.A. (ELMAR)

Kraft Foods España, S.A.

Cementos Tudela Veguín, S.A.

Miguélez, S.L.

Minero Siderúrgica de Ponferrada, S.A. (MSP)

Hullera Vasco-Leonesa, S.A.

Aceros Roldán, S.A.

Unión Minera del Norte, S.A.

e) Clasificación por áreas geográficas comarcales

Atendiendo a la configuración comarcal de León, las empresas de la muestra se han agrupado en las siguientes demarcaciones territoriales: 
LEÓN Y ÁREA METROPOLITANA (16)

Antibióticos, S.A. (León)

Alimentos Naturales, S.A. (León)

BA - Vidrio, S.A. (León)

Cristalera Industrial, S.A. (León)

Congelados y Derivados, S.A. (Elmar) (León)

Editorial Everest, S.A. (Trobajo del Camino)

Leches Maternizadas, S.A. (León)

Marmolería Leonesa, S.A. (León)

Miguélez, S.L. (Trobajo del Camino)

Rubiera, S.A. (León)

Tapicerías Soto, S.L. (Armunia)

Laboratorios SYVA, S.A. (Trobajo del Camino)

Laboratorios Ovejero, S.A. (Vilecha)

Manufacturas Teleno, S.L. (León)

Prefabricados de Cemento, S.A. (León)

Recuperación de Materiales Diversos, S.A. (León)

\section{EL BIERZO (9)}

Aceros Roldán, S.A. (Ponferrada)

Ferralla Eduardo e Hijos, S.A. (Cabañas Raras)

Señorío de Peñalba, S.A. (Toral de los Vados)

Comonor, S.A. (Cabañas Raras)

Cementos Cosmos, S.A. (Toral de los Vados)

Comercial Vinícola Villafranquina, S.A. (Villafranca del Bierzo)

Frigoríficos Industriales del Bierzo, S.A. (Friber) (Ponferrada)

Unión Minera del Norte, S.A. (Toreno y Torre del Bierzo)

Pizarras Expiz, S.A. (Puente de Domingo Flórez)

\section{TIERRAS DE LA BAÑEZA Y EL ÓRBIGO (7)}

Embutidos Rodríguez, S.L. (Soto de la Vega)

Legumbres Luengo, S.A. (La Bañeza)

Manufacturados de Aluminio, S.L. (La Bañeza)

Kraft Foods España, S.A. (Hospital de Orbigo)

Hijos de Antonio Gaspar Rosa, S.L. (La Bañeza)

Bañezana de Ferralla, S.L. (La Bañeza)

La Balinesa, S.L. (La Antigua) 


\section{LA MARAGATERÍA (2)}

Cecinas Pablo, S.A. (Astorga)

Plásticos del Noroeste, S.L. (Astorga)

LACIANA (1)

Minero Siderúrgica de Ponferrada, S.A. (Villablino)

GORDÓN (3)

Hullera Vasco-Leonesa, S.A. (La Robla y La Pola de Gordón)

Cementos Tudela Veguín, S.A. (La Robla)

Faminor, S.L. (La Robla)

\section{MONTAÑA DE RIAÑO Y CISTIERNA (2)}

Energía Viva, S.A. (Vidanes)

Selección de Productos Leoneses, S.A. (Vidanes)

\section{ALTO PORMA (2)}

Sociedad Española de Talcos, S.A. (Luzenac SET) (Boñar y Puebla de Lillo) Plantafarm, S.A. (Villanueva del Condado)

VALENCIA DE DON JUAN (4)

Cerámicas del Noroeste, S.A. (Valencia de D. Juan)

Gárnica Plywood, S.L. (Valencia de D. Juan)

Construcciones Metálicas Los Blancos, S.L. (Valencia de D. Juan)

Reposa - Lafon, S.A. (Villamañán)

\section{LA CABRERA (1)}

Pizarras La Baña, S.A. (La Baña)

PAYUELOS / TIERRA DE CAMPOS

Gordoncello, S.A. (Gordoncillo)

Industrias Lácteas Manzano, S.A. (Valderas)

Orica Explosivos Industriales, S. A. (Valderas) 


\section{DIAGNÓSTICO PATRIMONIAL, FINANCIERO Y ECONÓMICO}

\section{Estructura de la inversión empresarial}

Las inversiones a corto plazo, configuradas por las existencias de productos, los derechos de cobro frente a terceros, las inversiones financieras temporales y la tesorería, predominan dentro de la estructura de los activos empresariales. En 34 casos el activo circulante supera al nivel del activo fijo y en 16 se produce el caso contrario.

El liderazgo de las inversiones a largo plazo (inmovilizado) tiende a ser el predominante en las empresas de mayor tamaño (industria del carbón, química y cemento).

\section{Estructura de la financiación empresarial}

Protagonismo compartido entre recursos propios (RP) y recursos ajenos (RA). No hay un liderazgo claro en la procedencia del dinero que manejan las empresas. En 25 casos predominan los RP y en 25 los RA.

Cuando el protagonismo en la estructura financiera es de los recursos ajenos (RA) el nivel de endeudamiento duplica, en promedio, el tamaño de los recursos propios (RP).

Para el conjunto de la muestra, la hegemonía dentro de los recursos ajenos corresponde al endeudamiento a corto plazo, es decir, a las deudas con vencimientos no superiores a un año. La importancia financiera del endeudamiento a largo plazo es muy reducida, pues tan sólo en 4 empresas investigadas los fondos ajenos a largo plazo tienen más cuantía que las deudas a corto plazo. Este hecho caracteriza la temporalidad o estructura de los vencimientos de la financiación ajena.

El predominio del activo circulante dentro de la estructura de la inversión empresarial explica, por razones de equilibrio financiero (correlación entre naturaleza de la inversión y de la financiación), la reducida presencia de endeudamiento a largo plazo en los pasivos de los balances. Precisamente este rasgo del activo del balance favorece no tener que recurrir a la financiación ajena a largo plazo porque los recursos propios (suma del capital social más reservas) son suficientes para cubrir la inversión en inmovilizado.

Es destacable el moderado disfrute de financiación privilegiada (subvenciones de capital recibidas a fondo perdido para 
financiar infraestructura de producción o inmovilizado). Sólo 10 empresas analizadas trabajan con un nivel de subvenciones de capital igual 0 superior al $10 \%$ de su financiación total.

La dimensión de la empresa no es un factor que condicione la preferencia por los recursos propios o por los recursos ajenos, es decir, el tamaño del negocio no es un factor que influya de una manera determinante sobre la estrategia financiera seguida por las empresas.

\section{Distribución de beneficios}

Los beneficios tienen como principal destino su reinversión en la propia empresa. Es práctica mayoritaria (58\%) que los propietarios del negocio renuncien al reparto de dividendos con el fin de fortalecer la magnitud de los recursos propios.

De hecho, la formación de los recursos propios de las empresas se debe más a la transformación de los resultados positivos en reservas (autofinanciación), que a la inyección de nuevos fondos por parte de los accionistas (ampliaciones de capital).

\section{Garantía de acreedores}

En todos los casos analizados, y pese al nivel de endeudamiento con el que operan las empresas de la muestra, la totalidad de las mismas ofrece plena garantía de pago a sus acreedores, es decir, disponen de activo o inversión real suficiente para asegurar el reembolso o devolución de los capitales prestados.

Es de destacar que para la mitad de las empresas de la muestra el valor de los activos duplica el valor de las deudas contraídas.

El anterior resultado trasmite una imagen sólida de estabilidad patrimonial, dado que no existen motivos de intranquilidad para los aportantes de recursos ajenos. La solvencia a largo plazo está plenamente garantizada.

\section{Equilibrio financiero}

El test de adecuada correlación entre temporalidad de las inversiones y temporalidad de las financiaciones resulta satisfactorio para 
37 de las 50 empresas analizadas. Este resultado revela un alto grado de coherencia entre el ritmo de conversión en liquidez de las inversiones (activos) y los plazos o exigibilidad de las financiaciones (pasivos).

Cabe reseñar que en la mitad de los casos estudiados el volumen del capital circulante cubre la totalidad del saldo de las existencias de productos. Esta conclusión se concibe como un síntoma de estabilidad, robustez o firmeza financiera que favorece la solvencia empresarial a corto plazo.

Rentabilidad de las empresas (rentabilidad de la inversión o del negocio)

El rendimiento del capital invertido arroja resultados positivos en 48 (96\%) de los 50 casos estudiados.

La rentabilidad de las pequeñas y medianas empresas tiende a concentrarse en valores que no llegan a superar el umbral del 10\% (29 de 40 casos), mientras que en el caso de las empresas grandes su rentabilidad no supera este nivel en ninguno de los casos considerados. De hecho, ninguna gran empresa figura entre las diez más rentables. En consecuencia, los valores alcanzados por la rentabilidad de las diferentes actividades productivas no destacan o sobresalen por su magnitud.

De manera mayoritaria, los anteriores niveles de rentabilidad han sido generados por la actividad ordinaria o habitual desarrollada por las empresas, es decir, no han influido de manera significativa en el logro de estos umbrales de rentabilidad los resultados de naturaleza atípica o extraordinaria, caso de beneficios por enajenación de inmovilizado u otros similares.

En $2 / 3$ de las empresas investigadas se trabaja con márgenes de beneficios inferiores al $10 \%$, hecho que revela dificultad para abrir brecha entre ingresos y gastos. Síntoma propio de actuar en mercados caracterizados por un alto grado de competencia o rivalidad.

Las empresas pequeñas y medianas (PYMES) son las que dan un mayor giro o rotación al capital invertido al conseguir facturaciones anuales por un importe superior a su tamaño. Por el contrario, en las empresas grandes es más característico que 1 euro de inversión no logre generar 1 euro de ingresos. 
La rentabilidad de los negocios no entiende de tamaño o dimensión de los mismos. Pequeñas empresas obtienen rentabilidades mayores que las empresas medianas y grandes y a la inversa.

de la empresa)

Rentabilidad de los empresarios (rentabilidad del propietario

Para el $80 \%$ de la muestra, los niveles de rentabilidad conseguidos por los propietarios de las empresas superan la denominada rentabilidad de la despreocupación, es decir, logran cotas de rentabilidad mayores que las ofrecidas por inversiones alternativas sin riesgo, caso de las rentabilidades ofertadas por los títulos de la deuda pública a largo plazo o las imposiciones bancarias a plazo fijo (umbrales promedio del $3,5 \%-4 \%)$.

Consecuentemente, y por razones de rentabilidad, compensa ser empresario y asumir riesgos, diagnóstico que se puede considerar predominante en el conjunto de las empresas investigadas si se exceptúa la industria del carbón, actividad productiva muy dependiente para su continuidad de la recepción periódica de subvenciones a la explotación (ayudas al funcionamiento).

La tendencia de los análisis efectuados revela que las empresas obtienen de su endeudamiento una rentabilidad superior a su coste. Se encuentra en este aspecto un motivo para justificar el mayor peso que los recursos ajenos pueden llegar a tener en las estructuras financieras de los negocios, sin que ello suponga que las actividades productivas más endeudadas sean siempre las que reportan una mayor rentabilidad a sus propietarios.

Este comportamiento empresarial se ha visto favorecido por la rebaja gradual de tipos de interés que ha provocado la gestación del euro desde el año 1998.

\section{CONCLUSIONES}

1. Los recursos propios y el endeudamiento a corto plazo se erigen como los dos pilares básicos de la financiación empresarial, detectándose escasa predisposición por el uso de endeudamiento con exigibilidad superior a los doce meses. Esta característica de la estructura financiera se ha mantenido invariable a lo largo del bienio estudiado. 
2. La fortaleza de los recursos propios procede, fundamentalmente, de la reinversión de los beneficios en las propias empresas y no de ampliaciones de capital realizadas por los accionistas. Esta peculiaridad resta firmeza a la creencia generalizada de comportamientos especulativos por parte de los empresarios, pues de forma mayoritaria apuestan por no descapitalizar sus industrias al renunciar a un reparto prolongado en el tiempo de dividendos.

3. La financiación de las inversiones favorece la solvencia empresarial a corto y largo plazo, afianzando esta situación el equilibrio financiero de los negocios.

4. Como ya se puso de manifiesto en el informe del año 2001, son las empresas medianas, con plantillas comprendidas entre 51 y 250 trabajadores y cifras de negocio situadas entre 9 y 50 millones de euros, las que logran unos mayores niveles de rentabilidad.

5. Este formato empresarial entendemos que se concibe como el más idóneo para favorecer el renacimiento industrial de la economía leonesa, máxime cuando el avance tecnológico, el creciente e imparable grado de tecnificación de los procesos productivos, está requiriendo cada vez un menor número de trabajadores. Tiende a ser historia la creación de empresas industriales con mayúsculas plantillas (más de 1.000 trabajadores).

6. De manera mayoritaria la estructura financiera, la forma de financiar los negocios, ha influido de manera positiva sobre la rentabilidad obtenida por los propietarios de las empresas. De hecho, el coste del endeudamiento ha dejado de ser un castigo para la cuentas de resultados de las empresas.

7. En el marco de la coyuntura económica analizada, y por razones de rentabilidad, encuentra alicientes ser empresario o emprendedor y asumir riesgos en las diferentes ramas productivas consideradas salvo en la veterana industria del carbón por tratarse de rentabilidades subvencionadas. La incertidumbre que planea sobre la supervivencia de este todavía importante sector industrial continua sin despejarse, siendo ya peculiar de la gestión diaria del mismo funcionar en un clima de permanente reconversión.

8. Financiación y rentabilidad no son aspectos de la gestión de los negocios que guarden una relación directa con el tamaño de las empresas. Priman más circunstancias explicativas que se pueden vincular 
con la estructura de la propiedad (empresas familiares) y el grado de competencia existente en los diferentes mercados en los que se actúa.

9. El análisis patrimonial, financiero y económico realizado transmite, pese a las pérdidas observadas en cuatro de las empresas investigadas, una impresión positiva sobre el conjunto de las cincuenta actividades productivas objeto de estudio. Podemos emitir una opinión favorable en lo que respecta al mantenimiento y continuidad de los 8.362 empleos industriales recogidos en nuestra muestra y que se encuentran repartidos por toda la geografía leonesa, si bien hemos de dejar constancia de nuestra prudencia en lo que respecta a la ocupación encuadrada dentro de la industria del carbón (36\%), ya que se trata de una actividad cuya prolongación en el tiempo está sujeta a transcendentes resoluciones dictadas desde Bruselas y Madrid, tales como la fijación del volumen y emplazamiento de las denominadas "reservas estratégicas" y el Plan Nacional de Asignación de Emisiones Contaminantes derivado del cumplimiento del Protocolo de Kioto.

Respecto a las situaciones particulares de Antibióticos, S.A. y Enervisa en el momento de la emisión de este informe entendemos, como soluciones más factibles, la adquisición de la empresa química por empresas locales del mismo sector, así como la reconversión de la segunda en un modelo ganadero extensivo tradicional.

10. Como apunte adicional, y fuera del estricto marco del análisis contable, pero relacionado con el entorno y fortaleza del sector industrial leonés, tenemos que hacer mención a la dotación de nuevo suelo empresarial. Sin demérito hacia la consolidación y crecimiento de los polígonos industriales ya puestos en marcha, entendemos que resulta vital la agilización de cuatro proyectos que se encuentran en diferente grado de ejecución: polígono industrial de Villadangos del Páramo, polígono industrial de Cubillos del Sil, desbloqueo de la $2^{\mathrm{a}}$ fase del polígono de Onzonilla y parque tecnológico de León. Su culminación definitiva permitirá ampliar, sin lugar a dudas, la base industrial de la economía leonesa, fortaleciendo las infraestructuras tanto físicas como tecnológicas. Esto permitirá que las pequeñas y medianas empresas, con un peso relativo importante, puedan expandir sus negocios y no derivar en la deslocalización de las mismas hacia otras provincias con otros alicientes. 


\section{ANEXO DE RATIOS}

Endeudamiento: recursos ajenos / recursos propios

Poder de capitalización: reservas con origen en retención de beneficios / capital social desembolsado + prima de emisión

Garantía de acreedores: activo real / recursos ajenos

Estructura de la inversión: inmovilizado / activo real

Existencia de capital circulante: activo circulante / pasivo circulante

Grado de penetración del capital circulante: capital circulante / activo circulante

Cobertura de existencias: capital circulante / existencias

Rentabilidad de la empresa: beneficios antes de intereses e impuestos / activo real

Rentabilidad de la empresa ajustada: beneficios antes de intereses e impuestos \pm resultados extraordinarios / activo real

Margen de beneficios: beneficios antes de intereses e impuestos / ingresos ordinarios

Rotación del capital invertido: ingresos ordinarios / activo real

Rentabilidad del empresario: beneficio antes de impuestos / recursos propios

Apalancamiento financiero: rentabilidad del empresario / rentabilidad de la empresa 